\title{
Postexcitatory Inhibition of the Crayfish Lateral Giant Neuron: A Mechanism for Sensory Temporal Filtering
}

\author{
Eric T. Vu, Ari Berkowitz, and Franklin B. Krasne \\ Department of Psychology, Neuroscience Program, and the Brain Research Institute, University of California, \\ Los Angeles, California 90024
}

\begin{abstract}
Crayfish escape from threats by either giant neuron-mediated "reflex" tail flexions that occur with very little delay but do not allow for much sensory guidance of trajectory or by "nongiant" tail flexion responses that allow for sensory guidance but occur much less promptly. Thus, when a stimulus occurs, the nervous system must make a rapid assessment of whether to use the faster reflex system or the slower nongiant one. It does this on the basis of the abruptness of stimulus onset; only stimuli of very abrupt onset trigger giant-mediated responses. We report here that stimuli which excite the lateral giant (LG) command neurons for one form of reflex escape also produce a slightly
\end{abstract}

delayed postexcitatory inhibition (PEI) of the command neurons. As a result, only stimuli that become strong enough to excite the command neurons to firing threshold before the onset of PEI, within a few milliseconds of stimulus onset, can cause giant-mediated responses. This inhibition is directed to distal dendrites of the LG neurons, which allows for some location specificity of PEI within the sensory field of a single hemisegment.

Key words: postexcitatory inhibition; feed-forward inhibition; crayfish; lateral giant; escape; distal inhibition; dendritic integration; temporal filtering
Neurons in the CNS commonly inhibit, via local inhibitory interneurons, the same targets that they excite. Various roles have been postulated for such "feed-forward" inhibition (Freund and Antal, 1988; Turner, 1990; Pennartz and Kitai, 1991; Tomasulo et al., 1991). One consequence of feed-forward inhibition is that it suppresses excitation produced by all but the initial part of a period of stimulation, resulting in response selectivity for stimuli of abrupt onset over more slowly developing stimulation. We describe here evidence for feed-forward inhibitory circuitry that functions in this manner.

The crayfish lateral giant (LG) escape reflex is one of the few animal behaviors for which the outlines of a complete neural circuit have been described (see Fig. $1 A$ ). According to current belief, mechanosensitive primary afferents of the abdomen excite a group of sensory interneurons via cholinergic synapses, and both these interneurons and the primary afferents themselves innervate the LG command neurons via rectifying electrical synapses (Wine and Krasne, 1972; Krasne and Wine, 1987; Edwards et al., 1991; Miller et al., 1992). When the LGs reach firing threshold, they recruit the motor circuitry (shown in Figure 1A) that produces a lift of the hind end of the animal that tends to remove it from the source of disturbance. Another less studied pair of giant neurons, the medial giants (MGs) are recruited by

Received May 14, 1997; revised Aug. 21, 1997; accepted Aug. 28, 1997.

This research was supported by a National Science Foundation predoctoral fellowship to E.T.V., a National Institute of Mental Health Postdoctoral Fellowship to A.B., and National Institute of Neurological Disorders and Stroke Grant NS08108 to F.B.K. We thank Terri Teshiba for technical assistance and Dr. Donald Edwards for helpful discussions.

Correspondence should be addressed to Dr. Eric Vu, Division of Neurobiology, Barrow Neurological Institute, St. Joseph's Hospital and Medical Center, $350 \mathrm{~W}$

Thomas Road, Phoenix, AZ 85013.

Dr. Berkowitz's present address: Department of Zoology, University of Oklahoma, Norman, OK 73019.

Copyright (C) 1997 Society for Neuroscience $\quad 0270-6474 / 97 / 178867-13 \$ 05.00 / 0$ more rostral stimulation. MGs, via a motor circuit with slightly different connections, cause a backward directed flip.

A salient characteristic of both LG- and MG-mediated escape reactions is that they only occur in response to stimuli of very abrupt onset. Even massively tissue-damaging stimuli, if not abrupt, fail to evoke such escapes (Wine and Krasne, 1972). To some extent this temporal selectivity may be a product of the response characteristics of the primary afferents and sensory interneurons that provide input to the giant neurons (Wine, 1984; Krasne and Wine, 1987). However, we have noticed that the electrical EPSPs produced in the LGs by identified sensory interneurons become markedly diminished if they occur more than $\sim 4$ msec after the onset of a volley of sensory input. Furthermore, starting at the same time, the amplitude of the compound EPSP produced by the volley becomes quite sensitive to the membrane potential of the LG, getting smaller if the LG is depolarized by extrinsic current and larger if it is hyperpolarized. These observations are consistent with an increase in LG conductance and suggest the possibility that afferent activity might evoke chemical inhibitory input to the LGs along with electrical excitation.

An electron microscopic analysis of the LG dendrites and their inputs (Lee and Krasne, 1993) found evidence suggesting that both mechanosensory primary afferents and interneurons, en route to their LG dendritic targets, often form synapses on profiles containing synaptic vesicles that have the size and shape distributions characteristic of GABAergic inhibitors. These presumptive inhibitors in turn make synapses on LG dendrites. This evidence also suggests that excitation of the LGs may be closely followed by inhibition.

We establish here that excitation of the LGs is indeed followed by a period of postsynaptic inhibition mediated by $\mathrm{GABA}_{\mathrm{A}}$-like receptors. We also show that this "postexcitatory" inhibition (PEI) is generated in relatively distal parts of the LG dendrites, 
and we explore some functional consequences of this locus of action.

\section{MATERIALS AND METHODS}

Subjects. Crayfish (Procambarus clarkii) of both sexes measuring 6-11 cm from rostrum to telson were obtained from various local suppliers and maintained individually.

Anatomical background. The current view of the neural circuit afferent to the LG is diagrammed in Figure $1 A$. The classically described bilateral LG axons (Johnson, 1924) are actually chains of segmental neurons joined end to end by very efficient electrical "septal" junctions. They also communicate across the midline in each ganglion by electrical "commissural" junctions (Watanabe and Grundfest, 1961). Thus, the basic circuit shown in Figure $1 A$ is repeated on each side of each abdominal ganglion. Interneuron $\mathrm{A}$ is an identified sensory interneuron (Kennedy, 1971; Sigvardt et al., 1982) that fires only to input from ipsilateral sixth abdominal ganglion primary afferents, and it makes electrical synapses with the LG of each abdominal ganglion except the sixth. Figure $1 B$ is a schematic representation of the LG dendrites in a middle abdominal ganglion, indicating the location of their inputs. Input from terminals with elongated synaptic vesicles typical of GABAergic inhibitors are intermixed with excitors distally (relative to the axon) and also occur by themselves proximally (Lee and Krasne, 1993). The proximal inhibitors are presumed to mediate the recurrent inhibition that is produced by the firing of the LGs or MGs and prevents the giants from firing in the middle of a previously begun tail flip (Roberts, 1968; Vu and Krasne, 1992; Vu et al., 1993). Distal inhibitors are presumed to be involved in descending tonic inhibition of LG escape, which lowers the probability of escape in various behavioral circumstances (Krasne and Wine, 1975; Krasne and Lee, 1988; Vu et al., 1993; Krasne and Teshiba, 1995); we will argue that distal inhibitors also mediate the PEI studied here.

Experimental preparation. Animals were gradually cooled to approximately $5^{\circ} \mathrm{C}$ before dissection was started. The abdomen was then separated from the thorax and pinned dorsal side up in a Sylgard-lined
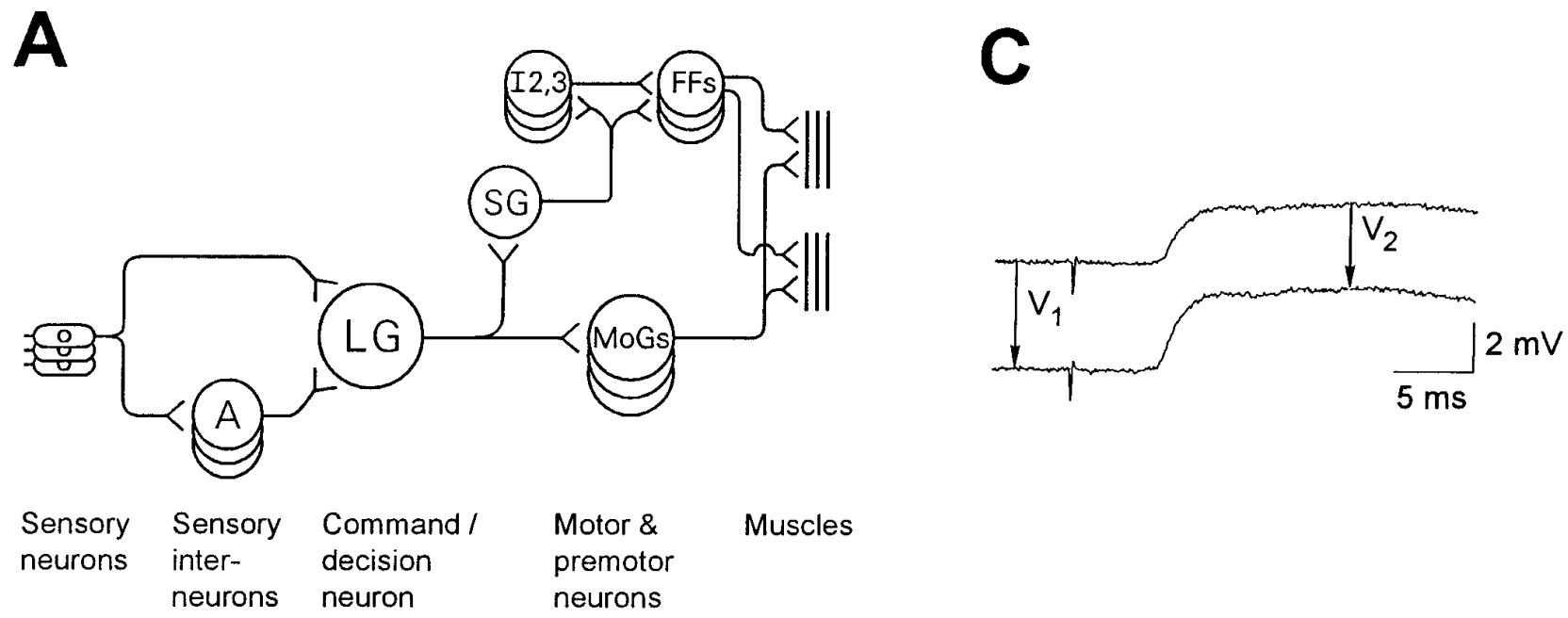

B

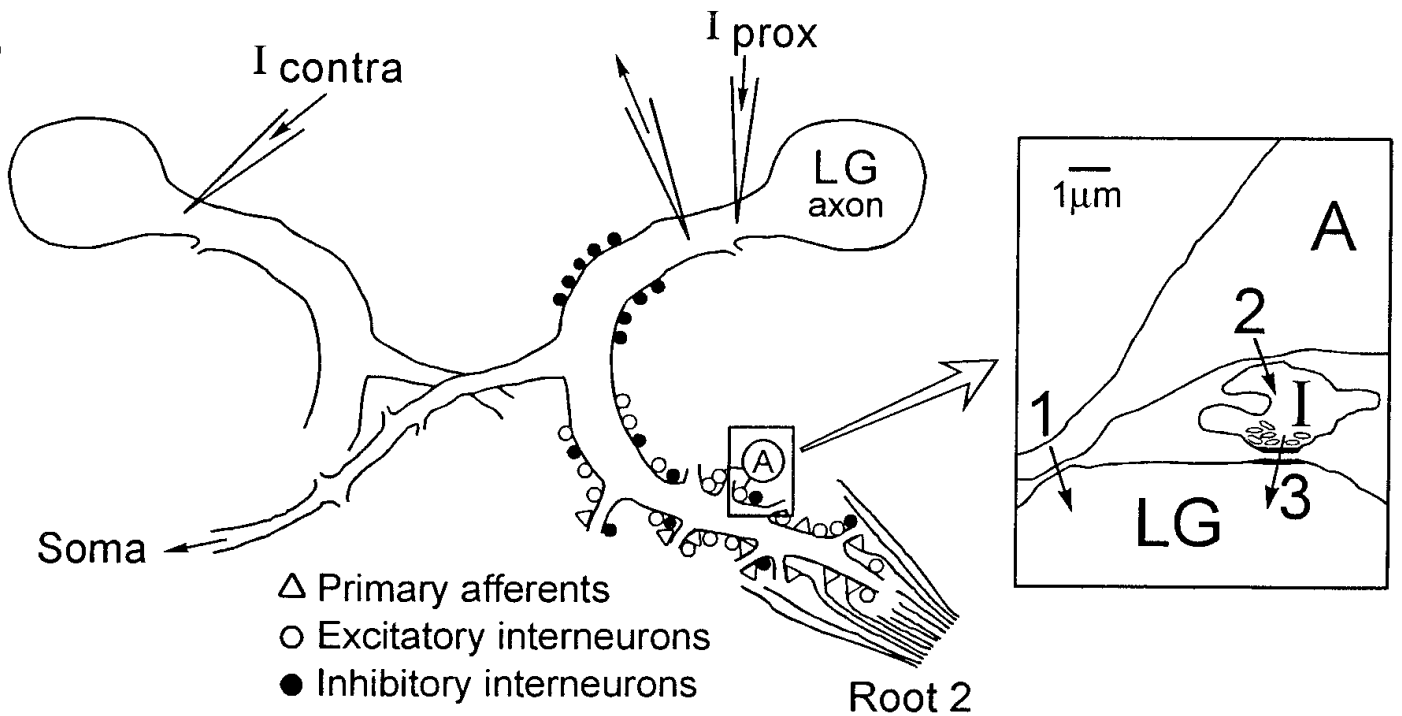

Figure 1. Anatomical background and methods. $A$, Schematic diagram of the neural circuit for LG escape reactions. $A$, Interneuron A. $L G$, Lateral giant; $S G$, segmental giant; $M o G s$, motor giants; $I 2,3$, intersegmental neurons 2 and $3 ; F F s$, fast flexor motoneurons. $B$, Diagram of LG dendrites as seen in transverse section (dorsal up), showing the location of anatomically classified synaptic inputs to LG (Lee and Krasne, 1993) and the sites of microelectrode placement. The bilateral LG neurons are electrically coupled by commissural synapses near the midline of the ganglion; part of the contralateral LG neuron is drawn. $I_{\text {prox }}$, Current injected into the initial segment of the axon, proximal to the recording electrode; $I_{\text {contra }}$, current injected into the contralateral LG. Inset (right) is a diagram based on electron micrographs (Lee and Krasne, 1993) of a region of contact between interneuron A and an LG dendrite. 1, A presumed excitatory synapse between interneuron A and LG. 2, Synapse between interneuron A and a GABAergic inhibitory neuron $(I)$. 3, Synapse between inhibitory neuron and LG dendrite. $C$, Definition of voltage changes used to compute percentage reduction of $V_{\text {prox }}$ and $V_{\text {contra }}$ in LG during a synaptic potential. Shown is a depolarizing recurrent inhibitory IPSP produced by stimulating MG while LG is at normal resting potential (top) and during hyperpolarization of LG with injected current (bottom). 
Plexiglas chamber (volume $=20 \mathrm{ml}$ ). The terga and gut were removed, and the flexor musculature was separated at the midline and pinned apart to expose the ventral nerve cord (Krasne, 1969). Motor roots were cut to eliminate movements. If two microelectrodes were to be inserted, the sheath overlying the rostral-dorsal portion of either the third or fourth abdominal ganglion was surgically removed. Picrotoxin (Sigma) or $\mathrm{Cd}^{2+}$ Ringer's solution $\left(1 \mathrm{mM} \mathrm{Ca}^{2+}\right.$ replaced by $\left.\mathrm{Cd}^{2+}\right)$ was delivered to some preparations via a cannula inserted into the rostral stump of the ventral artery that vascularizes the abdominal CNS. The methods for cannulation and drug delivery were as described in Miller et al. (1992) and based on the methods of Mulloney et al. (1987). Briefly, the cannula was attached to a four-way valve that received inputs from elevated reservoirs that were subjected to equal air pressure. The entire preparation was immersed in chilled $\left(14-18^{\circ} \mathrm{C}\right.$ ), aerated Ringer's solution (composition as in Van Harreveld, 1936).

Stimulation and recording. Single-barrel microelectrodes filled with 2.5 M potassium acetate or $3 \mathrm{M} \mathrm{KCl}$ and having 10-30 $\mathrm{M} \Omega$ resistances were used to impale the LG just rostral to the septal junction, i.e., at the axon initial segment (Krasne, 1969). A pair of platinum hook electrodes was used to stimulate primary afferent fibers in the ipsilateral second root of the ganglion under study (either the third or fourth abdominal ganglion). Ipsilateral first root electrodes were additionally used in some experiments. To evoke recurrent inhibition, the ipsilateral MG axon occasionally was stimulated directly via a fine pin electrode insulated to very near its tip. The axon of interneuron A was also stimulated directly with a focal pin electrode placed ventral to a hemiconnective ipsilateral to the impaled LG. All electrical stimuli to roots and connectives were $0.1 \mathrm{msec}$ monophasic pulses; all constant current injections into the LG were at least $60 \mathrm{msec}$ long. Root stimulations were spaced at least $1.5 \mathrm{~min}$ apart (except as noted) to minimize the well known depression of transmission in the afferent pathway to the LG that is responsible for behavioral habituation (Krasne, 1969; Zucker, 1972). Stimulating and recording equipment were standard. Signals were digitized at $25 \mathrm{KHz}$ per channel by a Metrabyte DAS-20 board and an IBM PC.

Experimental procedures. In each experiment we compared the magnitude of a voltage change evoked in LG at rest with that of the voltage change evoked in the same way during the course of a postsynaptic potential (PSP), either the second root-evoked PSP or the depolarizing IPSP evoked by firing of the medial giant axon (Roberts, 1968). Three types of voltage change were studied: (1) the unitary electrical EPSP produced by an ipsilateral interneuron $\mathrm{A}$ action potential $\left(V_{\mathrm{A}}\right)$ (see Fig. $1 A) ;(2)$ the voltage change produced by a constant current injected into the initial segment of the LG axon, which we call $V_{\text {prox }}$ because it is generated and measured proximal to the axon initial segment; and (3) the voltage change produced in the LG axon initial segment by a constant current injected into the electrically coupled contralateral LG $\left(V_{\text {contra }}\right)$. The voltage changes $V_{\text {prox }}$ and $V_{\text {contra }}$ were produced by a second, current-injecting microelectrode inserted into the LG axon initial segment either ipsilaterally (within 0.2 space constants on average in electrotonic distance from the recording microelectrode) or contralaterally.

To quantify the effects of synaptic activity on the voltage changes produced by injected test currents, we determined the voltage change (at its plateau) produced by a constant current in the absence of evoked synaptic activity $\left(V_{1}\right)$ (see Fig. $1 C$ ) and the voltage change produced by the same current during synaptic activity $\left(V_{2}\right)$. For the latter measurement, current injection was begun, and membrane potential was allowed to plateau before synaptic activity was evoked; $V_{2}$ was measured as the difference between the voltage produced by current injection during synaptic action and the voltage during synaptic action alone. The percentage reduction of the voltage change produced by the injected current, $100 \times\left(V_{1}-V_{2}\right) / V_{1}$, provided a measure of the effect of synaptic action. Percentage reductions were calculated for each time point during the PSP, yielding a continuous curve of percentage reduction. Normally, percentage reduction curves were determined at least four times and averaged.

Percentage reductions in voltage changes determined in this way are equal to percentage decreases in input resistance, insofar as synaptically produced resistance changes are slow enough so that capacitative effects can be ignored. Thus, the percentage reduction curves approximate continuous estimates of $L G$ input resistance change throughout the course of the PSP. These estimates are most accurate for the slowest changing portion of the PSP, which occurs after the $\alpha$ and $\beta$ peaks (see below).

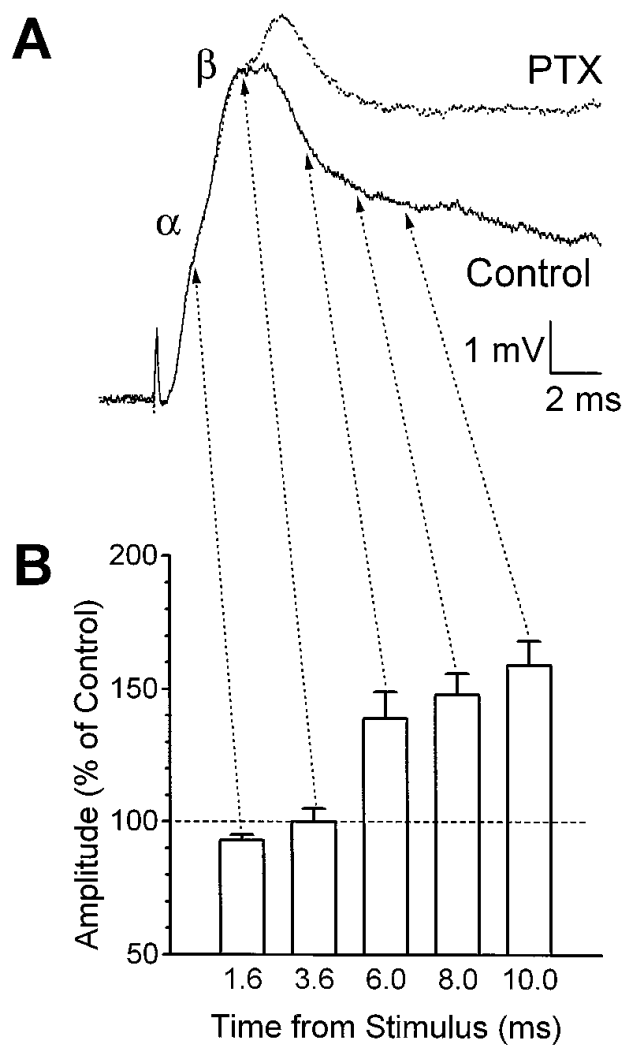

Figure 2. Effect of picrotoxin (PTX) on the shape of the root-evoked PSP recorded in LG. $A$, Superimposed traces from the same LG neuron before and during application of $7.5 \mu \mathrm{M}$ PTX. $B$, Mean amplitude of the PSP from eight animals at successive times after the stimulus, after PTX application. The PSP amplitude at each time point is expressed as a percentage of its amplitude before PTX application. The dose of PTX varied between animals $(7-10 \mu \mathrm{M})$. Arrows to $A$ point to the equivalent times in the traces of one animal.

\section{RESULTS}

\section{Evidence for PEI of the LG neuron during sensory excitation}

Sensory-evoked PSPs recorded in LG are sensitive to picrotoxin (PTX)

When mechanosensory primary afferent fibers of a segmental sensory root are electrically stimulated, a multiphasic PSP is recorded intracellularly from the LG neuron of that segment (see Fig. $2 A$, control trace) (Krasne, 1969). The first $(\alpha)$ component of this "root-evoked PSP" is produced by monosynaptic excitatory input from the primary afferents, whereas the second $(\beta)$ and later components are thought to consist of EPSPs produced by repetitive firing of mechanosensory interneurons that were excited by the primary afferents (Fig. $1 A$ ). To evaluate the possibility that these primary afferents also activate GABAergic inhibitory input to the LG, we examined the effect of PTX, which blocks $\mathrm{GABA}_{\mathrm{A}}$ receptor-coupled chloride channels. PTX applied at doses of 7-10 $\mu \mathrm{M}$ did not change the peak of the $\alpha$ or the initial part of the $\beta$ components significantly but did reduce the extent to which the PSPs dropped after the time of the control PSP $\beta$ peak, in eight out of eight preparations (Fig. $2 B$ ); in some PTXpoisoned preparations the PSP continued to rise after the time of the pre-PTX $\beta$ peak (Fig. $2 A$ ). These observations suggest re- 
Figure 3. Reduction of an electrical EPSP when evoked during a root-evoked PSP. $A$, Top trace, Root-evoked PSP alone; bottom trace, unitary EPSP $\left(V_{\mathrm{A}}\right)$ evoked by action potential in interneuron A alone; middle trace, $V_{\mathrm{A}}$ evoked during a root-evoked PSP (solid trace), superimposed on the rootevoked EPSP alone (dashed trace). Arrows point to the peak of $V_{\mathrm{A}}$. Calibrations apply to all traces. $B$, The mean percentage reduction of the $V_{\mathrm{A}}$ peak when occurring during a root-evoked PSP (as compared with $V_{\mathrm{A}}$ occurring alone) at various times after the root stimulus ( filled circles). Error bars represent SEM for 10 animals; some data points without error bars were obtained from only one animal. The typical time course of the root-evoked PSP is illustrated by the dashed curve.
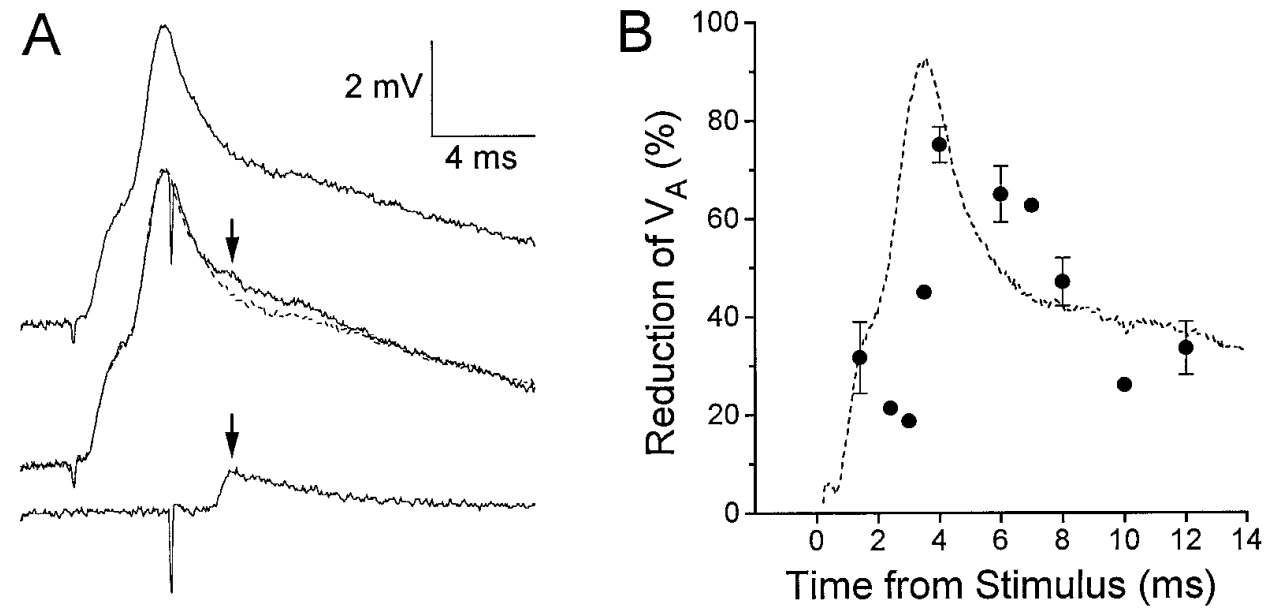

lease from a GABAergic inhibition that begins at about the time of the normal peak of $\beta$.

Lower doses of PTX had qualitatively similar effects, but to a lesser degree, whereas higher doses reduced the $\beta$ peak significantly, possibly via nonspecific effects on the cholinergic transmission between primary afferents and sensory interneurons (Marder and Paupardin-Tritsch, 1980; Miller et al., 1992).

\section{Monosynaptic test EPSPs do not summate linearly with the root-evoked PSP}

If the LGs are postsynaptically inhibited after the peak of the $\beta$ component, then EPSPs occurring at this time should be reduced in amplitude. We tested this possibility by electrically stimulating the axon of interneuron A (Zucker, 1972) (Fig. 3A, bottom trace) so that its monosynaptic (electrical) EPSP would occur during the root-evoked EPSP. Interneuron A is not fired by stimulation of the third or fourth segment sensory roots, so we could be assured that it fired only when stimulated directly and it would not be rendered refractory during the compound EPSP.

Figure $3 A$ illustrates the result of evoking an interneuron $\mathrm{A}$ EPSP $\left(V_{\mathrm{A}}\right)$ during the root-evoked PSP. The top trace is the root-evoked PSP by itself, and the bottom trace is $V_{\mathrm{A}}$ alone. When $V_{\mathrm{A}}$ was evoked during the root-evoked PSP (middle trace), $V_{\mathrm{A}}$ was noticeably reduced (arrow above trace). In Figure $3 B$, this reduction is expressed as a percentage of the amplitude of the unattenuated $V_{\mathrm{A}}$ (see Materials and Methods for procedures for signal averaging and calculations) at various times during the root-evoked PSP. Maximal reduction seemed to occur during the most rapidly falling portion of the $\beta$ component. In ten different preparations, $V_{\mathrm{A}}$ was reduced by an average of $47.1 \pm 4.9 \%$ (SEM) when timed to occur at $8.0 \mathrm{msec}$ after the root stimulus.

\section{LG conductance increases during root-evoked PSPS}

The shape of the root-evoked PSP changed readily with imposed shifts of the LG membrane potential. Hyperpolarization of the LG caused the late phase of the PSP to increase, whereas depolarization caused it to decrease in amplitude (Fig. 4B). The $\alpha$ and $\beta$ components usually changed in a similar direction but to a much lesser extent. The relative sensitivity of the post- $\beta$ components to membrane potential alteration is consistent with the presence of a postsynaptic conductance associated with a reversal potential relatively near the resting level (see below). This suggests a postsynaptic inhibitory action.

Postsynaptic conductance increases during root-evoked PSPs were evaluated by looking for decreases of the voltage $\left(V_{\text {prox }}\right)$ produced by injecting a constant hyperpolarizing current pulse (resulting in hyperpolarization of $3.1-8.6 \mathrm{mV}$ from rest) into the initial segment of the LG during the PSP. The percentage reduction of $V_{\text {prox }}$ is shown in Figure $4 C$ (solid curve), with the PSP from the preparation also shown for reference (dashed curve). A small reduction was seen during the $\alpha$ and $\beta$ components, whereas a more prominent reduction occurred during the "late phase" of the PSP, starting immediately after the peak of the $\beta$ component. It seemed likely that the late phase reduction might be attributable in part to an inhibitory conductance increase in LG, and our focus in the following sections will be on exploring its properties. The basis of the early reduction in $V_{\text {prox }}$ is probably different and is addressed in Discussion.

The voltage $\left(V_{\text {contra }}\right)$ produced by injecting current into the contralateral LG was also reduced during root-evoked PSPs. The time course was similar to that for $V_{\text {prox }}$, but the extent was greater (Fig. 4D). The reasons for this difference will be discussed below, but because $V_{\text {contra }}$ provides a much more sensitive measure of the shunting caused by PEI than does $V_{\text {prox }}$, it was often used as the single measure of PEI in this study.

\section{Conductance increase during late phase PSP is sensitive to PTX}

Although the increase in magnitude of the late phase of the root-evoked PSP in the presence of PTX is consistent with the presence of postsynaptic inhibition of the LGs during this period, PTX should antagonize stimulus-driven inhibition of transmission between primary afferents and interneurons (Kennedy et al., 1974, 1980; Kirk and Wine, 1984; Kirk, 1985). Thus, an unknown portion of the increase in root-evoked PSP size could be attributable to increased excitatory drive from disinhibited sensory interneurons. We therefore evaluated the effect of PTX on several indicators of inhibition that cannot be explained in this way.

Using the experimental paradigm of Figure 3, we measured the percentage reduction of $V_{\mathrm{A}}$ at $8 \mathrm{msec}$ after the root stimulus in three preparations exposed to PTX. Whereas under control conditions $V_{\mathrm{A}}$ was reduced by an average of $47.1 \pm 4.9 \%(n=10)$, in $7 \mu \mathrm{M}$ PTX it was reduced by only $18.2 \pm 5.4 \%$ (Fig. $5 B)(n=3)$. Only partial washout of PTX antagonism was possible, and this took an extremely long time ( $\sim 3-4 \mathrm{hr}$ half-time of recovery) (Kennedy et al., 1980; Vu and Krasne, 1993). Neither the LG resting potential nor its resting input resistance was affected by this concentration of PTX.

PTX also decreased the reduction of $V_{\text {contra }}$ (and $V_{\text {prox }}$ ) seen during the late phase of root-evoked PSPs (Fig. 6), indicating that 

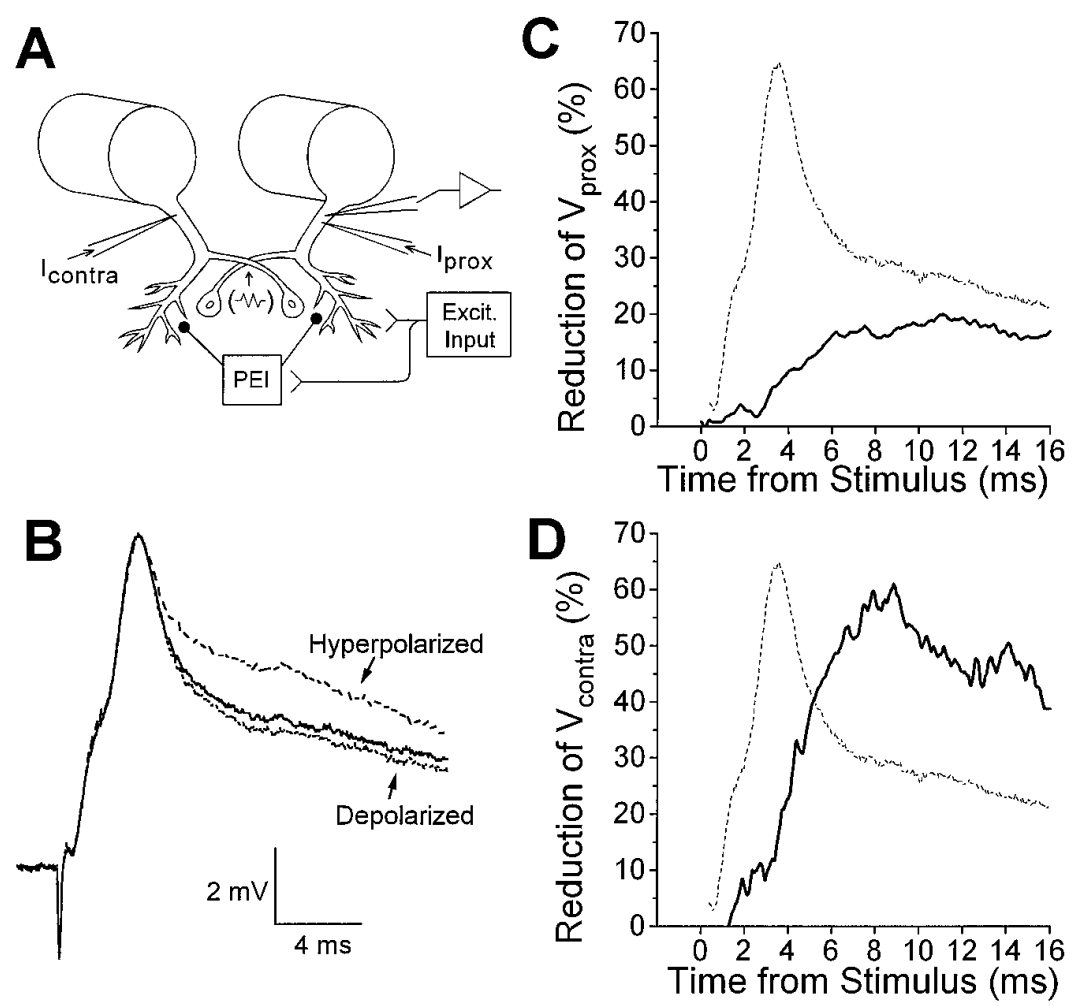

Figure 4. Shunting of currents injected into LG during the root-evoked PSP. A, Diagram of experimental setup: voltage measurements were obtained from one LG neuron; $V_{\text {prox }}$ was produced by $I_{\text {prox }}, V_{\text {contra }}$ by $I_{\text {contra }}$. PEI, Postexcitatory inhibition; Excit. Input, excitatory input. The commissural junction is represented by the resistor symbol at the point of overlap of the two LGs. $B$, Superimposed are root-evoked PSPs recorded at normal resting potential (middle trace), while the LG was hyperpolarized by $7.8 \mathrm{mV}$ (top trace), and while it was depolarized by $0.9 \mathrm{mV}$ (bottom trace). $C$, Mean percentage reduction of $V_{\text {prox }}$ over the time course of the root-evoked PSP for one animal (solid curve). $D$, Mean percentage reduction of $V_{\text {contra }}$ over the time course of the root-evoked PSP for one animal (solid curve). In $C$ and $D$, the dashed curve represents the typical time course of the root-evoked PSP. Data in $B, C$, and $D$ are from three different preparations.

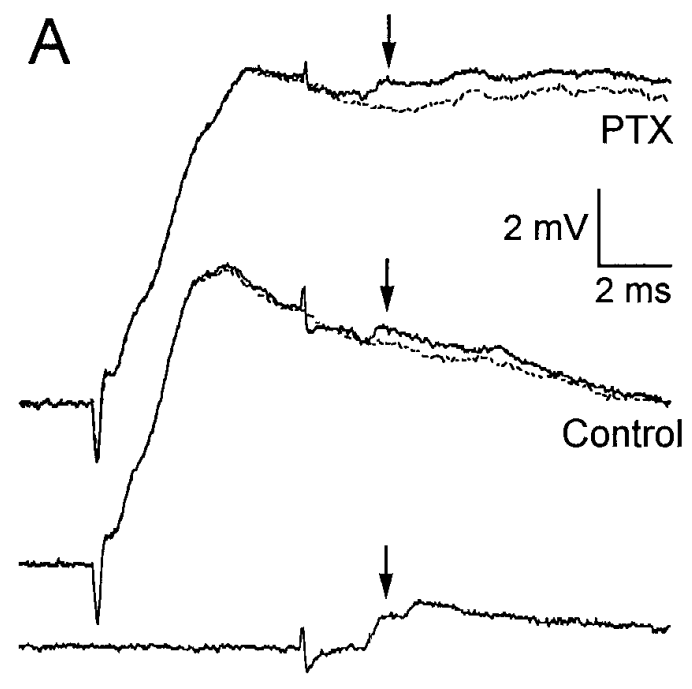

Figure 5. Effect of PTX on the reduction of $V_{\mathrm{A}}$ by the root-evoked PSP. $A$, The bottom trace shows $V_{\mathrm{A}}$ evoked in the absence of root stimulation. (The second "hump" in the trace, which was not considered for measurement, was a slightly later unitary EPSP associated with the firing of another interneuron.) The middle two traces are $V_{\mathrm{A}}$ evoked during a root-evoked PSP (solid trace), superimposed on a root-evoked PSP alone (dashed trace); both middle traces were obtained in the absence of PTX. The top two traces are the same as the middle two traces except that they were obtained in the presence of $7 \mu \mathrm{M}$ PTX. All traces were recorded from the same animal. The arrows point to the peak of $V_{\mathrm{A}}$ in each case. $B$, Mean percentage reduction of $V_{\mathrm{A}} \pm$ SEM when its peak was timed to occur $8.0 \mathrm{msec}$ after the root stimulus, as in the example shown in $A$, in the absence (control; $n=10$ animals) and presence of PTX $(n=3)$. the conductance change responsible for the reduction is decreased. However, PTX seemed to have little effect on the reductions of $V_{\text {contra }}$ and $V_{\text {prox }}$ that occur before the peak of the $\beta$ component or beyond $12-14 \mathrm{msec}$ after the root stimulus (see Discussion).

As mentioned above, higher doses of PTX could not be used to attempt to abolish completely the conductance increase during the late phase of the root-evoked PSP. However, the amount by which 7-10 $\mu \mathrm{M}$ PTX decreased this conductance change was similar to the amount of decrease of MG-evoked recurrent inhibition of LG (Roberts, 1968) in the same preparations (not shown). This suggests that higher doses of PTX would antagonize PEI even further, because recurrent inhibition can be abolished by high doses of
PTX and thus is thought to be mediated entirely by $\mathrm{GABA}_{\mathrm{A}}$ receptors (Krasne and Roberts, 1967; Vu and Krasne, 1993).

\section{Reversal potential during PEI is inconsistent with excitation}

Attempts to assess the reversal potential for PEI are complicated by two factors. (1) PEI is always mixed with concurrent distally originating excitation, and (2) as demonstrated below, the inhibitory input responsible for PEI is localized distally, at quite a distance from the recording and current-passing electrodes used to assess reversal potentials.

The amplitude of the root-evoked PSP at $8 \mathrm{msec}$ from the stimulus was measured as a function of membrane potential, which was controlled by injection of a constant current into the 

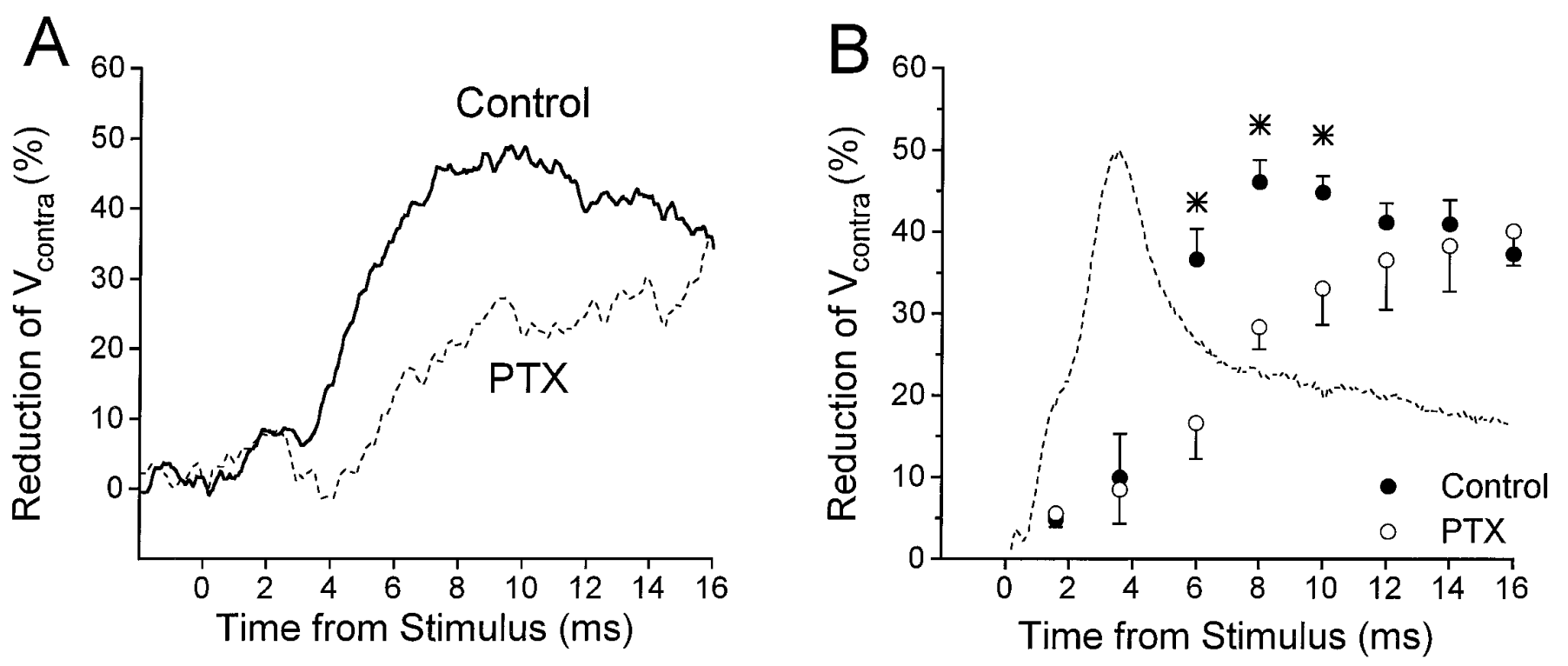

Figure 6. PTX decreases shunting of test currents by the root-evoked PSP. $A$, Mean percentage reduction of $V_{\text {contra }}$ over the time course of the root-evoked PSP for one animal, in the absence (solid curve) and presence (dashed curve) of PTX. $B$, Mean percentage reduction of $V_{\text {contra }}$ at successive times after root stimulation, in the absence ( filled circles; $n=7$ preparations) and presence (open circles; $n=4$ ) of PTX. Asterisks indicate those time points for which $V_{\text {contra }}$ reduction was significantly different before and during PTX application. The typical time course of the root-evoked PSP is illustrated by the dashed curve.

Figure 7. Estimated reversal potential of PEI. $A$, Filled circles plot amplitude of root-evoked PSP at time of maximum PEI ( $8 \mathrm{msec}$ after root shock) as a function of membrane potential $\left(E_{m}\right)$, controlled by current injection through a second electrode. Least squares linear fit extrapolates to $-58 \mathrm{mV}$ at zero PSP amplitude. For comparison, open squares show amplitude of $\beta$ peak, and open circles show peak amplitude of recurrent IPSP evoked by stimulation of MG. $E_{r}$, Resting potential. $B$, Estimated reversal potentials from multiple preparations. Rec. Inhib., Recurrent inhibition.
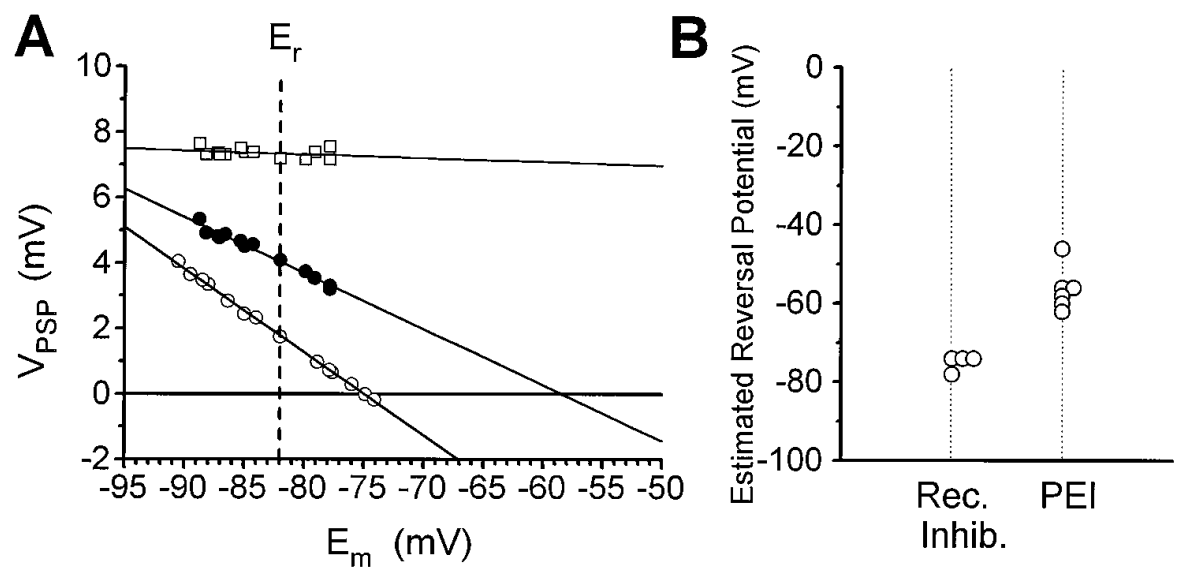

LG. Results from one preparation are illustrated in Figure $7 A$. The extrapolated reversal potential in this experiment was -58 $\mathrm{mV}$, and the average for six preparations (Fig. $7 B$ ) was $-56.0 \pm$ $2.4 \mathrm{mV}$. Reversal potentials for chemical or electrical EPSPs are expected to be near or above zero, and measured reversal potentials should be even more positive relative to the resting potential when measurements are made with current-passing and recording electrodes distant from sites of synaptic input, as in the present case. Therefore, the present values support the hypothesis that the PSP at $8 \mathrm{msec}$ is in part a chemical IPSP (possible complications associated with rectifying electrical transmission are deferred to Discussion). For comparison, we found that the reversal potential for the MG-evoked recurrent IPSP was $-75 \mathrm{mV}$ in the preparation illustrated, and the mean for four preparations (Fig. 7B) was $-75.1 \pm 1.1 \mathrm{mV}$ (cf. Roberts, 1968).

The amplitudes of the $\alpha$ and $\beta$ components of the EPSP also varied somewhat with membrane potential but were much less sensitive than the late phase of the EPSP (Fig. $7 A$ illustrates the amplitude of $\beta$ as a function of membrane potential). This is consistent with our belief (see Discussion) that the reductions of $V_{\mathrm{A}}, V_{\text {prox }}$, and $V_{\text {contra }}$ that occur during the $\alpha$ and $\beta$ components reflect a cellular mechanism different from PEI.

\section{Distal locus of PEI}

The above data strongly suggest the existence of a PTX-sensitive form of inhibition of the LGs, driven by sensory activity. In this section, we present evidence that in contrast to recurrent inhibition, which acts near the axon initial segment, PEI acts distally in the LG dendrites.

\section{PEI reduces $V_{A}$ more than it reduces $V_{\text {prox }}$}

Comparison of Figures $3 B$ and $4 C$ indicates that whereas both $V_{\mathrm{A}}$ and $V_{\text {prox }}$ are reduced after the peak of $\beta$, suggesting an increased conductance of the LGs, $V_{\mathrm{A}}$ is reduced approximately three times as much as $V_{\text {prox. }}$ In contrast, Roberts (1968) found that EPSPs and $V_{\text {prox }}$ were attenuated comparably during recurrent inhibition of the LGs. These observations suggest that the inhibitory synapses that produce recurrent inhibition cause a conductance 
A

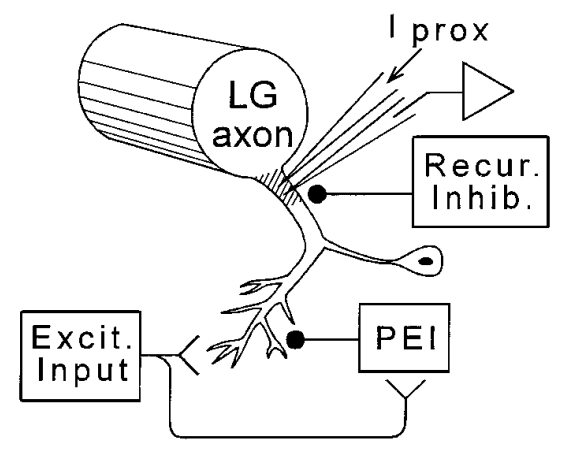

C
B
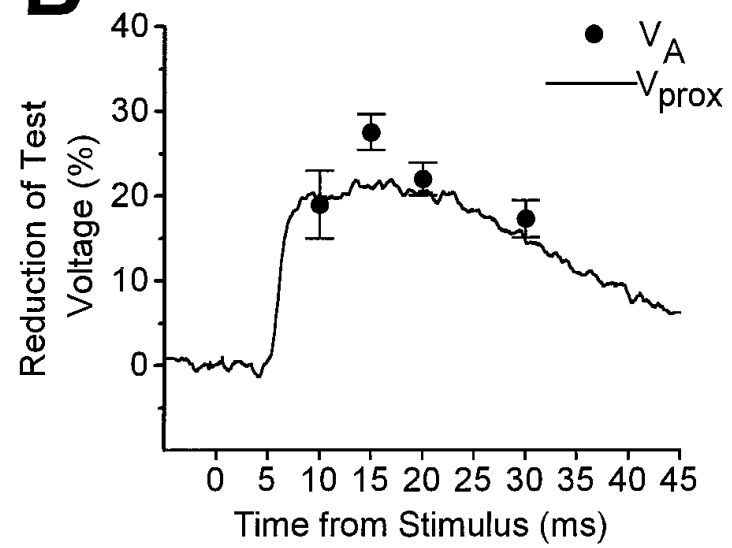
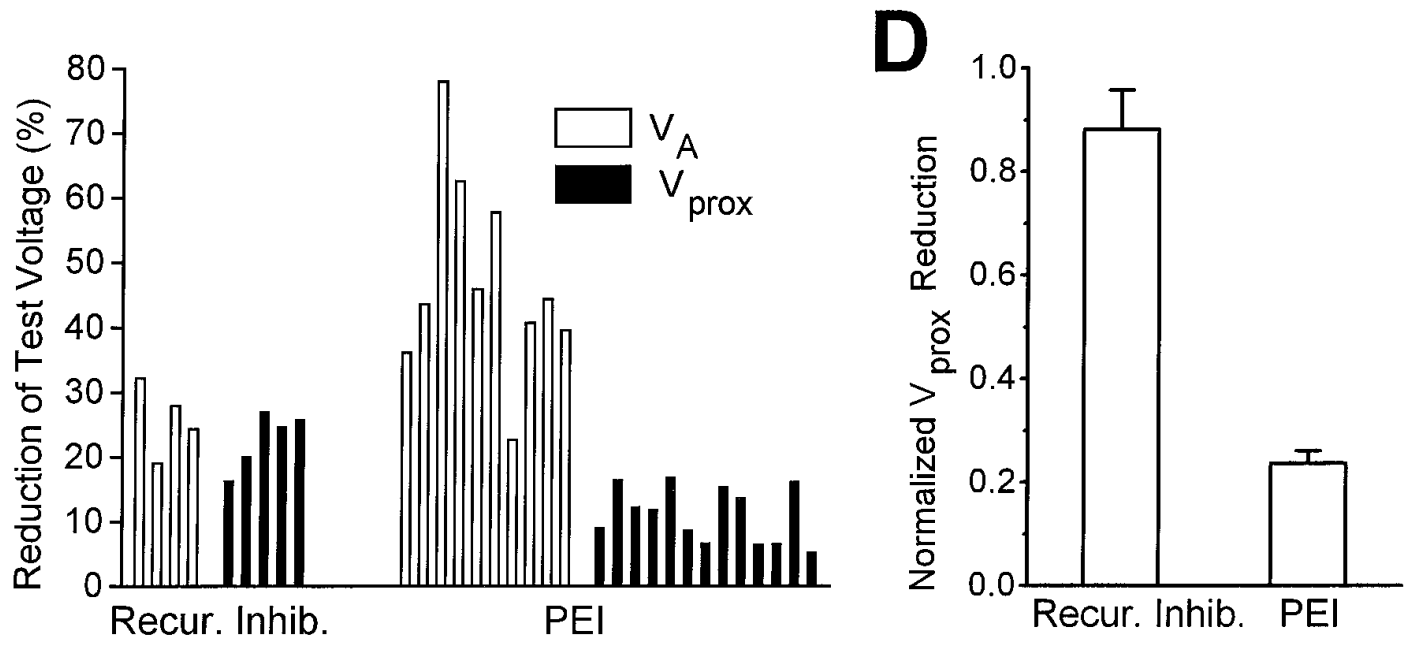

Figure 8. Different cellular loci of PEI and recurrent inhibition. $A$, Diagram of experimental setup: voltage measurements were made at the base of the main dendrite, near the spike-initiating zone of the LG axon. $V_{\text {prox }}$ was produced by $I_{\text {prox }} . V_{\mathrm{A}}$ is generated by synapses in relatively distal LG dendrites (Lee and Krasne, 1993). The locus of action of recurrent inhibition is thought to be near the main dendrite and our recording site (Roberts, 1968; Vu et al., 1993). The presumptive locus of PEI, on distal dendrites, is indicated. $B$, Mean reduction by recurrent inhibition of $V_{\mathrm{A}}\left(\right.$ filled circles) and of $V_{\text {prox }}$ (solid curve) at various time points, for one animal. Recurrent inhibition was evoked by an action potential in the MG (Roberts, 1968). C, Comparison of the reduction of $V_{\mathrm{A}}$ (open bars) and $V_{\text {prox }}$ (filled bars) by recurrent inhibition and PEI. Each bar represents the mean value obtained from one preparation (at $8.0 \mathrm{msec}$ after root stimulation for PEI and at $10 \mathrm{msec}$ after MG stimulation for recurrent inhibition). $D$, Mean reduction of $V_{\text {prox }}$ across animals as a fraction of the mean reduction of $V_{\mathrm{A}}$, calculated from data in $C$. Recur. Inhib., Recurrent inhibition; Excit. Input, excitatory input.

increase localized near the recording electrode in the initial segment of the LG axon, so that synaptic current originating in distal dendrites and current injected from a nearby microelectrode are shunted equally well as they flow toward the recording site. In contrast, the synapses mediating PEI might produce a conductance increase at locations sufficiently distal in the LG dendrites so that synaptic currents are shunted readily but axonally injected test currents are shunted poorly (Fig. 8A).

To examine systematically the difference between the two types of inhibition, we measured the percentage reductions of $V_{\mathrm{A}}$ and $V_{\text {prox }}$ for both PEI and recurrent inhibition in a number of preparations (Fig. 8). It is apparent from Figure $8 B-D$ that for recurrent inhibition $V_{\text {prox }}$ and $V_{\mathrm{A}}$ were attenuated to a similar extent, whereas for PEI $V_{\text {prox }}$ was attenuated much less than $V_{\mathrm{A}}$. The ratio (percentage reduction of $\left.V_{\text {prox }}\right) /\left(\right.$ percentage reduction of $V_{\mathrm{A}}$ ) for recurrent inhibition $(0.88 \pm 0.08)$ was significantly different $(p<$ 0.01 ; two-tailed $t$ test) from that for PEI $(0.24 \pm 0.03)$.

\section{Additional evidence for the distal locus of PEI}

If PEI is mediated by synapses on distal LG dendrites, then it might reduce a voltage change produced at the recording electrode by a current injected into the electrically coupled contralateral LG ( $V_{\text {contra }}$ ) more than it would $V_{\text {prox }}$ (which is produced by proximally injected current), because the current path from contralateral injection site to recording site might include distal membrane regions with activated inhibitory conductances (Fig. 9A). Figure 9C (right) shows that $V_{\text {contra }}$ is indeed reduced much more than $V_{\text {prox}}$.

To some extent, a greater reduction of $V_{\text {contra }}$ than $V_{\text {prox }}$ would be expected even for strictly proximal but bilateral inhibition, because a contralaterally originating current would be shunted both near its injection site and on the side of recording, whereas an ipsilaterally originating current would be shunted mainly near its origin. If inhibition were strictly proximal, the fractional drop in voltage $\Delta V_{\text {contra }} / V_{\text {contra }}$ would be about twice $\Delta V_{\text {prox }} / V_{\text {prox }}$ if the inhibition were as great contralaterally to a stimulated root as on the side of stimulation, and it would be somewhat less otherwise (see Appendix and Fig. 9B). As seen in Figure 9C, however, $\Delta V_{\text {contra }} / V_{\text {contra }}$ is significantly greater than twice $\Delta V_{\text {prox }} / V_{\text {prox }}$ $(p<0.001)$, which implies that PEI is not entirely proximal.

Our data allowed us to ask, incidentally, whether recurrent inhibition, which is known to operate proximally, also affects 

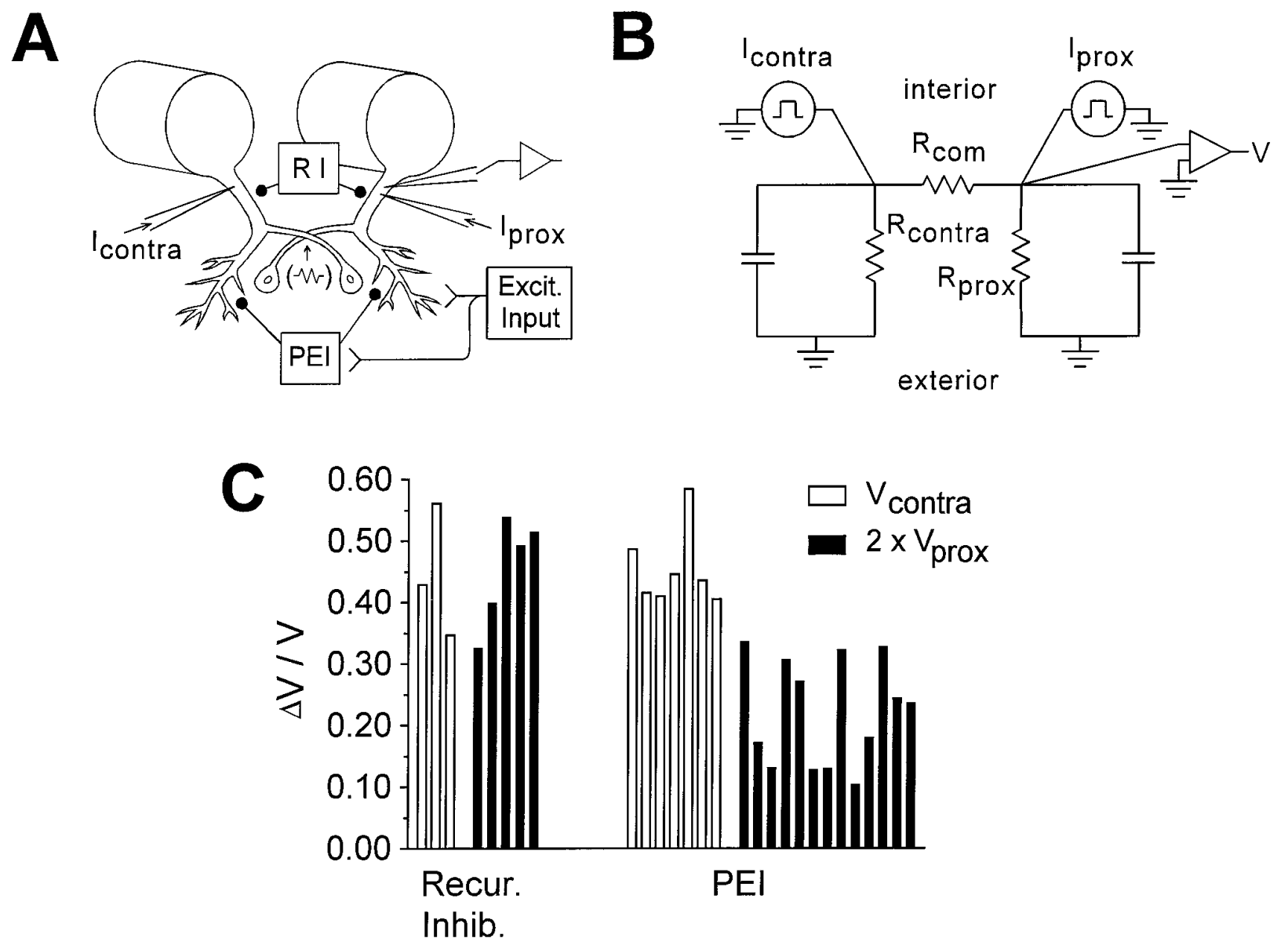

Figure 9. Additional evidence for the different cellular loci of PEI and recurrent inhibition. $A$, Diagram of experimental arrangement. $B$, Linear circuit model used to predict relative effects of an exclusively proximal, bilateral inhibition on $V_{\text {contra }}$ and $V_{\text {prox }}$ (Vu et al., 1993). Equivalent batteries for the resting potential were omitted. See Appendix for description of circuit components. $C$, Comparison of the proportion of reduction of $V_{\text {contra }}$ (open bars) and twice the reduction of $V_{\text {prox }}$ ( filled bars) by recurrent inhibition and PEI (see Results for rationale). Each bar represents the mean value obtained from one preparation. Excit. Input, Excitatory input; Recur. Inhib., recurrent inhibition; RI, recurrent inhibition.

distal dendrites. Because recurrent inhibition acts bilaterally and symmetrically (Roberts, 1968), $\Delta V_{\text {contra }} / V_{\text {contra }}$ should be twice $\Delta V_{\text {prox }} / V_{\text {prox }}$ if the inhibition is purely proximal. As seen in Figure $9 C$ (left), this is the case.

\section{Relation between PEI and preceding excitation}

The electron microscopic data (Lee and Krasne, 1993) reviewed in the introductory remarks suggest that PEI may be mediated by local circuit excitation of inhibitory neurons by neurons that also excite the LGs, with the result that GABA is released onto the LGs near the point of their excitation. The results of the previous section indicating that PEI acts on distal dendrites, where excitatory synapses occur (Lee and Krasne, 1993), is consistent with this anatomical conjecture. One consequence of this arrangement is that the greater the degree of LG afferent activity, the greater should be the amount of PEI. Also, if mechanosensory input to the LG dendrites is topographic, inhibition produced by stimulation of a particular bodily location might preferentially counteract excitatory input from the same or nearby locations. The next two sections evaluate these possibilities.

\section{Recruitment of PEI with stronger excitation}

Figure 10 indicates that as the strength of root stimulation was progressively increased, PEI-assessed by attenuation of $V_{\text {contra }}$ (as well as $V_{\text {prox }}$ and $V_{\mathrm{A}}$; not shown)-increased roughly in parallel with the amplitude of the root-evoked PSP. In general, low stimulus levels evoked small PSPs without evoking measurable PEI. Additionally, there was no discernible PEI after the unitary EPSP produced by a single action potential in interneuron A (not shown). Together, these findings suggest either that inhibitors must receive a threshold amount of excitation before releasing GABA or that a substantial amount of release must occur before its effects can be detected.

\section{Site specificity of PEI}

To test for spatial selectivity of PEI, we evoked PEI by stimulating one sensory root and then delivered a second stimulus to test the selectivity of PEI; the second stimulus was delivered either to the same sensory root or to a root having a segmental sensory field different from that of the first root. The monosynaptic, presumably electrical $\alpha$ component of the second PSP was timed to occur during maximum PEI, approximately $8 \mathrm{msec}$ after the first stimulus. Figure $11 A$ illustrates the basic experimental design. Figure $11 A_{1}$ shows, superimposed, the PSPs produced by a root 1 stimulus alone and by the same stimulus followed by a second root 1 stimulus; also shown (filled circles) is the expected response to the second stimulus in the absence of PEI. In this case the $\alpha$ compo- 

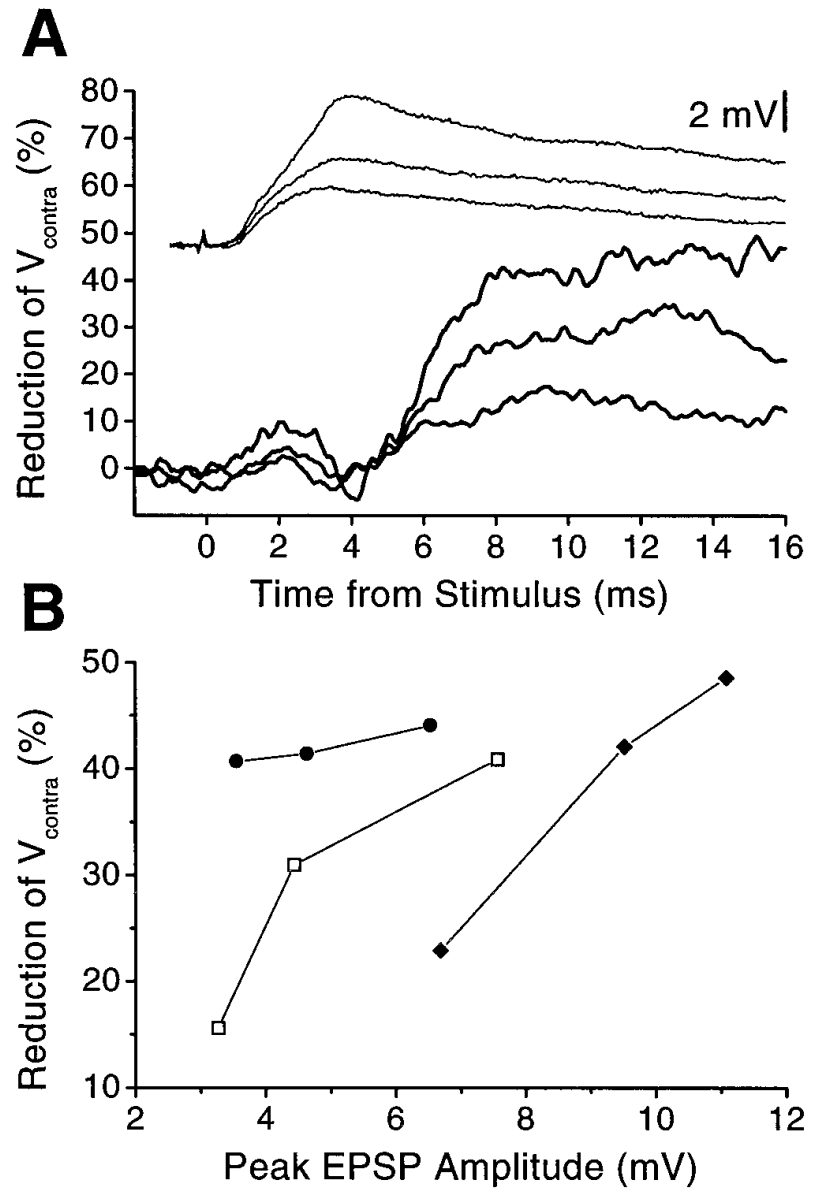

Figure 10. Graded recruitment of PEI. A, Time course of reduction of $V_{\text {contra }}$ in one preparation for three strengths of root stimulation (lower, thick curves; corresponding PSPs are shown as thin curves above). Stronger stimuli produced more reduction of $V_{\text {contra. }} B$, Percentage reduction of $V_{\text {contra }}$ as a function of peak amplitude of the root-evoked PSP for three animals. EPSP amplitudes that did not reduce $V_{\text {contra }}$ significantly are not shown.

nent of the second PSP was attenuated to a small fraction of its control amplitude. Figure $11 A_{2}$ shows that when the second stimulus was delivered to root 2 instead, the $\alpha$ component was only slightly reduced. Thus, the PEI produced by stimulating a sensory root was more effective in reducing responses to subsequent stimulation of that same root than to stimulation of a different one. Figure $11 B$ shows a similar pattern in the same preparation when PEI was evoked via root 2 instead. Figure $11 C$ summarizes results from examination of the PEI produced by root 1 in 8 and root 2 in 10 preparations $(p<0.001$ for $t$ test of difference between percentage reduction for same-root vs different-root test stimuli).

The possibility must be considered that responses to test stimuli were attenuated more when the two stimuli were delivered to the same root simply because the excitatory synapses that mediate the root-evoked PSP were fatigued at $8 \mathrm{msec}$ after a previous stimulation. This seemed unlikely, because Zucker (1972) had shown that the presumably electrical $\alpha$ component could follow $200 \mathrm{~Hz}$ root stimulation without decrement. Nevertheless, to confirm the absence of synaptic fatigue we assessed the attenuation of the second response to pairs of root shocks $8 \mathrm{msec}$ apart in preparations in which an attempt was made to abolish PEI by blocking chemical synaptic transmission with $1 \mathrm{mM} \mathrm{Cd}^{2+}$ Ringer's solution. The sample traces in Figure $11 D$ indicate that in the absence of PEI, the second response to a pair of root shocks to a single root is not attenuated significantly. However, $\mathrm{Cd}^{2+}$ Ringer's solution slightly reduced the $\alpha$ component, even when evoked at low frequencies, to an average value of 0.88 times its original size (Fig. 11E, open circles). This raises the possibility that $\alpha$ itself might be mediated partially by chemical transmission, and that the greater reduction of $\alpha$ in same-root compared with differentroot tests was attributable to fatigue of this small chemical contribution to the $\alpha$ component.

If this were the case, then in same-root tests $\alpha$ would be attenuated by a factor attributable to PEI, which would be the same as the factor by which $\alpha$ is attenuated in different-root tests, and additionally by a factor attributable to fatigue. Therefore, the attenuation attributable to fatigue could be estimated from the ratio of same-root to different-root attenuations; these estimates are plotted as filled circles in Figure $11 E$. We then compared these values to the attenuation of $\alpha$ caused by $\mathrm{Cd}^{2+}$, which provided an estimate of the attenuation that would occur if the chemical contribution to $\alpha$ were removed completely as a result of fatigue (Fig. 11E, open circles). This comparison shows that the additional factor by which $\alpha$ is reduced on same-root tests is on average more than twice what could be accounted for even by the full fatigue of the chemical contribution to $\alpha(p<0.03$, likelihood ratio test; $t$ test avoided because of unequal group variances). Thus, the greater reduction of $\alpha$ seen in same-root tests is attributable to actual differences in the strength of PEI between the two conditions.

\section{DISCUSSION}

The findings reported here establish that activity in abdominal sensory roots results in both excitation and inhibition of the LGs, with the inhibition slightly delayed relative to the excitation. The existence of this PEI necessitates a revision of the neural circuit for LG escape (Fig. 12A). Anatomical studies suggest that similar feed-forward inhibitory relationships are common in nervous systems (Shepherd, 1990); however, because the behavioral functions of the present circuit are so well known, it is possible in this case to discern the functional consequences of the inhibition.

The major role that we ascribe to PEI is the discounting of excitatory input to the LGs that is delayed relative to the onset of sensory stimulation, with the result that the LGs fire only for stimuli that become strong rapidly. PEI thus helps explain, along with sensory accommodation (Wine, 1984; Krasne and Wine, 1987) and other factors discussed below, why LG escape occurs only in response to stimuli of abrupt onset and not to gradually increasing stimuli, even though they may become tissue-damaging.

LG and MG escape reactions provide the advantage of short latency but the disadvantage of stereotyped form, escape trajectories being either symmetrically upward (LG) or symmetrically backward (MG) (Wine and Krasne, 1972; Krasne and Wine, 1984). Crayfish also have more flexible nongiant tail flexion escape circuitry, which is used to produce a range of not necessarily symmetrical, visually guided escapes (Wine and Krasne, 1972; Krasne and Wine, 1984) that have much longer latencies. Consequently, on being stimulated, the nervous system must make a rapid decision about whether to use the $\mathrm{LG} / \mathrm{MG}$ system or to wait and allow the slower but more sophisticated nongiant system to generate the response. This decision is based on the abruptness of 

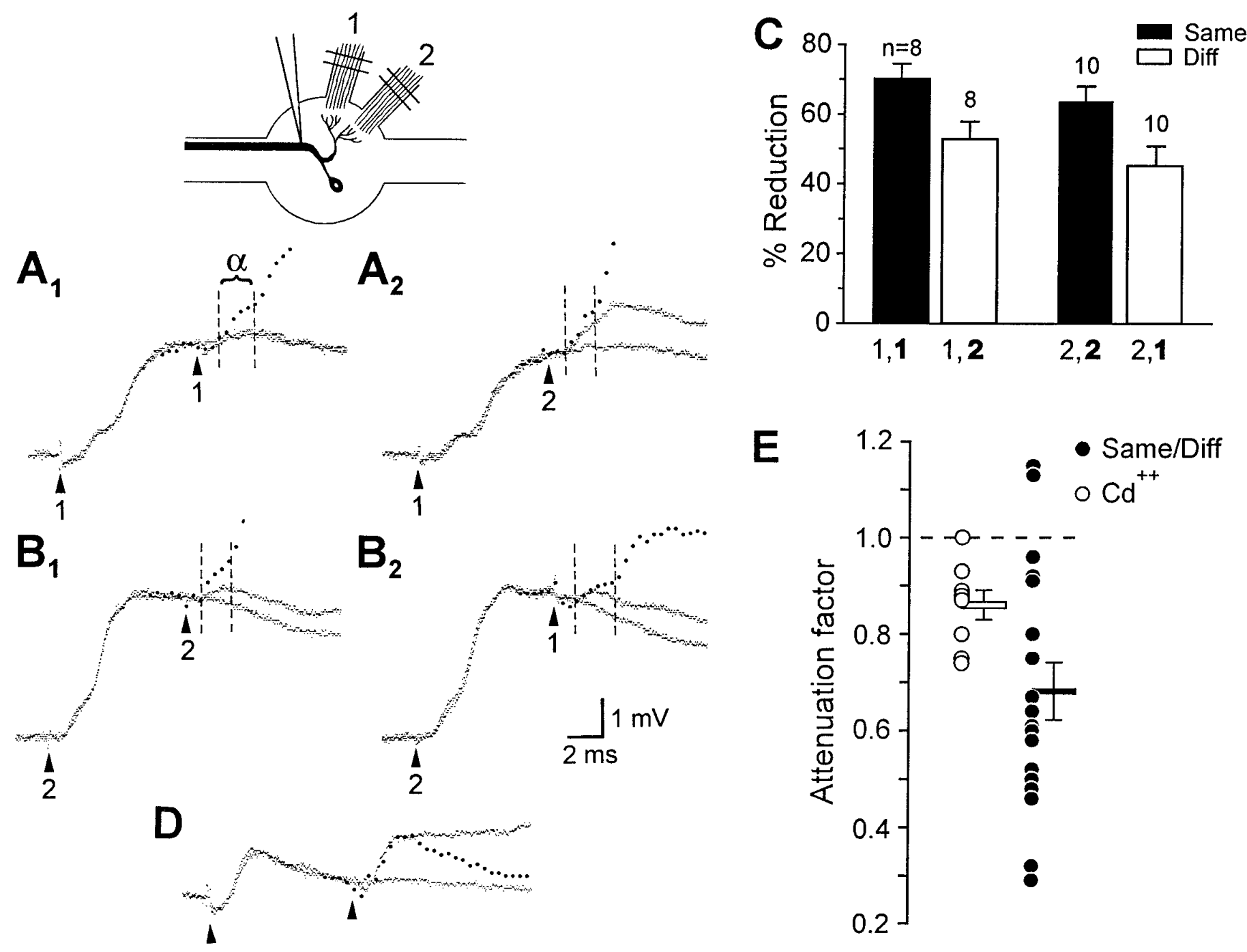

Figure 11. Site specificity of PEI. A, B, Top left, Experimental arrangement. In each case, the response in LG to a pair of sensory root stimuli, 8 msec apart, is superimposed on the response to the first stimulus alone. The filled circles plot the calculated sum of the response to the first stimulus alone and the second stimulus alone, with the second response delayed $8 \mathrm{msec}$ from the first. The dashed lines demarcate the duration of the $\alpha$ component of the response to the second stimulus. $A_{1}$, First stimulus: root 1 ; second stimulus: root 1 (note that the second stimulus produced almost no response). $A_{2}$, First stimulus: root 1; second stimulus: root 2. $B_{1}$, First stimulus: root 2; second stimulus: root 2. $B_{2}$, First stimulus: root 2; second stimulus: root 1 . $C$, Mean percentage reductions of $\alpha$ component amplitudes of responses to second stimuli. For each bar, the second stimulated root, which produced the measured response $($ bold $)$, and the first stimulated root (lighter font) are indicated. The number of roots examined and SEM are indicated. $D$, Cd ${ }^{2+} \mathrm{Ringer}^{2} \mathrm{~s}$ solution. Superimposed responses to root 2 stimulated once and stimulated twice with an $8 \mathrm{msec}$ inter-stimulus interval. The filled circles plot the calculated sum of the response to root 2 alone and the same response offset by $8 \mathrm{msec}$. E, Filled circles, Ratio of same-root to different-root $\alpha$ component

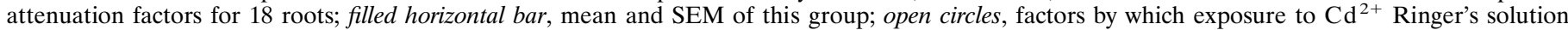
attenuated $\alpha$ component amplitude in eight preparations (see Results for explanation); open bar, corresponding mean and SEM. Diff, Different.

stimulus onset, with PEI and other factors being responsible for assessing abruptness.

PEI also limits the maximum excitation that abrupt stimuli produce in the LGs. Therefore, its up- or downregulation in principle could regulate reflex excitability, as suggested for Aplysia protective reflexes (Fischer and Carew, 1993, 1995; Trudeau and Castellucci, 1993a,b). We have explored, but have not found evidence for, the possibility that tonic inhibition (Krasne and Wine, 1975; Vu et al., 1993) might be mediated by an upregulation of PEI (E. T. Vu and F. B. Krasne, unpublished observations).

\section{Additional mechanisms causing EPSP reduction}

PEI is unlikely to be the only mechanism responsible for reductions of test voltages during root-evoked PSPs, because PTX has little effect on reductions that occur before the peak of the $\beta$ component or more than $\sim 14 \mathrm{msec}$ after the stimulus (Fig. 6). Study of synaptic transmission to the LGs, as well as to other neurons in the escape circuit, provides evidence for several other mechanisms that are likely to be involved.

Most or all excitation of the LGs is via rectifying electrical synapses (Edwards et al., 1991). These junctions become (bidirectionally) conductive when their presynaptic elements become positive to their postsynaptic dendritic targets (i.e., "forwardbiased") by some threshold voltage; in the best studied cases, they also remain conductive for $\sim 1 \mathrm{msec}$ after the presynaptic potential falls below this threshold (Jaslove and Brink, 1986; Giaume et al., 1987). These properties have two important consequences. (1) 

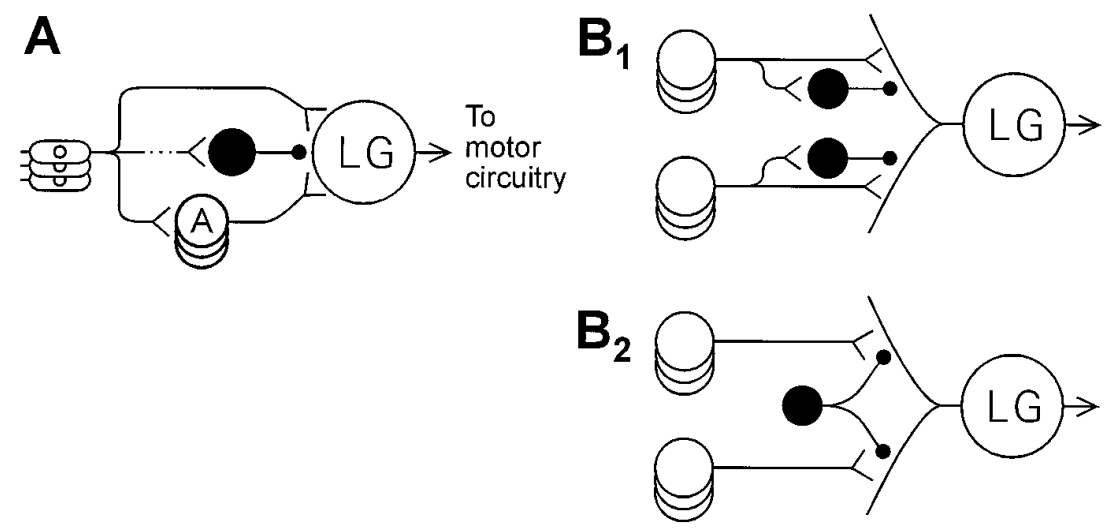

Figure 12. Revision of afferent portion of LG reflex circuit. $A$, An inhibitory neuron (black) has been added to circuit. $B$, Possible arrangements to account for site specificity of PEI (see Discussion).

For the period after completion of a presynaptic spike during which the synapse remains conductive, the presynaptic compartment provides a current sink for the LG dendrites that hastens the discharge of dendritic capacitance, and thus the decay of the EPSP, and shunts any additional EPSPs generated by nearby synapses during the period of increased junctional conductance. We refer to this as presynaptic shunting. Such shunting would be expected to be most important immediately after the initial volley of sensory interneuron firing that produces the rise of the $\beta$ component. (2) Impulses arriving at a terminal on an already depolarized LG dendrite will not forward-bias the junction to an opening point until the presynaptic action potential has sufficiently exceeded the potential of the postsynaptic dendrite. Therefore, current will flow to the LG only during a portion of the presynaptic spike, and it will be driven by a potential difference that is less than the amplitude of the presynaptic spike. For both reasons, the EPSP amplitude will be reduced. We refer to these effects as voltage-dependent attenuations of synaptic efficacy.

Both presynaptic shunting and voltage-dependent attenuation potentially contributed to reductions of $V_{\mathrm{A}}$ in our experiments. Furthermore, even in the absence of PEI, voltage-dependent attenuation would cause EPSPs mediated by rectifying junctions to be reduced by exogenous depolarization, and insofar as postsynaptic hyperpolarization causes increases in the duration of suprathreshold forward-biasing, it should enlarge EPSPs. The resulting EPSP modulation by postsynaptic membrane potential would give the impression of an increased postsynaptic conductance, and in our experiments such modulation would be seen as a reduction in the voltage produced by an injected constant current $\left(V_{2}\right.$ in Fig. $1 C$ ), as if the applied current were shunted by PEI.

Finally, shunting of synaptic potentials can result from the fact that the conductance of the LG axon tends to increase when it is depolarized by more than $\sim 10 \mathrm{mV}$ (Edwards, 1990), presumably because of the opening of voltage-dependent potassium channels (delayed rectification). We discuss below the contribution of all these effects.

\section{Contributions to temporal filtering}

Sensory temporal filtering is partly mediated by properties of primary afferents and sensory interneurons (Wine, 1984; Krasne and Wine, 1987). However, of the temporal filtering mechanisms operating at the level of the LGs that were discussed above, what is the relative contribution of PEI?

The relatively small reduction of $V_{\mathrm{A}}$ that occurs during the $\alpha$ component is probably caused by presynaptic shunting or voltagedependent attenuation, because this reduction is insensitive to PTX and there is probably not sufficient time for release of inhibitory transmitter at that point of the PSP. However, it is the large reductions that occur after the peak of the $\beta$ component that are probably of greatest functional importance in preventing the LG from firing to stimuli of gradual onset.

Presynaptic shunting, voltage-dependent attenuation, and PEI probably all contribute to the reductions measured 4-14 msec after a root stimulus. Presynaptic shunting would be greatest immediately after the synchronous volley of input that produces $\beta$. Voltage-dependent attenuation would be greatest when the LG dendrites are most depolarized, but it is difficult to determine the potential of distal dendrites. Edwards et al. (1991) found that EPSPs are reduced by $\sim 1 \%$ per millivolt of postsynaptic depolarization. Thus, if dendritic potentials after the $\beta$ peak were five times greater than those at the initial segment, they would be $10-25 \mathrm{mV}$ and should produce a $10-25 \%$ (PTX-insensitive) attenuation of EPSPs. In our experiments, attenuations at $8 \mathrm{msec}$ were $23-78 \%$ ( $47 \pm 15.5 \%$ SD). PEI presumably accounts for at least half of this attenuation because PTX reduced it by half. Moreover, a significant amount of PEI probably remained, because $7 \mu \mathrm{M}$ PTX also removed only about half of the recurrent inhibition of the LGs, which is likely to be mediated by the same type of GABA receptors as PEI.

At $14-16$ msec after a sensory root shock, $V_{\mathrm{A}}, V_{\text {prox }}$, and $V_{\text {contra }}$ were all attenuated $20-40 \%$ by a mechanism that is apparently insensitive to PTX. However, because PTX augments depolarization of the $\mathrm{LG}$ at this time (Fig. 2A) (Krasne and Roberts, 1967), presumably by increasing sensory interneuron activity (Kennedy et al., 1974, 1980; Kirk and Wine, 1984), presynaptic shunting and voltage-dependent forms of attenuation should also be increased by the PTX. Thus, the fact that PTX did not increase attenuation at $14-16 \mathrm{msec}$ suggests that a substantial amount of PEI exists after $14 \mathrm{msec}$ and that PTX reduced it while augmenting presynaptic shunting and voltage-dependent attenuation, leading to a negligible net change.

\section{Significance of the distal locus of PEI}

PEI seems to be directed to the distal, branching region of the LG dendrites, which is also where excitatory inputs synapse (Lee and Krasne, 1993), rather than to the initial segment, where firing of the LGs could be prevented more efficiently. Like tonic inhibition of the LGs, which reduces the excitability of escape in a restrained crayfish and is also focused distally, PEI would be expected to reduce the likelihood of $\mathrm{LG}$ escape without absolutely preventing it ( $\mathrm{Vu}$ and Krasne, 1992; Vu et al., 1993).

The distal locus of PEI makes it possible for localized sensory inputs to evoke PEI in a particular region of the LG dendritic 
tree. If excitatory and inhibitory input originating from a given region of the body converge on a common dendritic region, then PEI that prevents response to gradually increasing stimulation at one location within a segment would not necessarily prevent responses to sudden stimulation at a different location. In fact, PEI produced by stimulating either the first root of a segment, which innervates ventral skin and swimmerets, or the second root, which innervates dorsal exoskeleton, reduces later excitation evoked by stimulation of the same root more than it does excitation produced by stimulation of the other root. Additionally, PEI evoked by stimulating a given root reduces excitation produced by later stimulation of the same root more than it does excitation produced by later stimulation of contralateral roots or roots of other segments ( $\mathrm{Vu}$ and Krasne, unpublished observations), although this does not require distal inhibition.

Although the degree of site-specificity of PEI remains to be determined, the neural architecture that gives rise to the specificity is a matter of some interest. Are differentially inhibitable portions of the dendritic tree serviced by different inhibitors (Fig. $12 B_{1}$ ), or is PEI recruited by local circuit action, with different LG excitors causing transmitter release from different branches of one inhibitory neuron (Fig. 12B ${ }_{2}$ ) (Lee and Krasne, 1993)? Also, one wonders whether descending modulation of escape reflex excitability by means of tonic inhibition might be exerted via the same distally projecting inhibitory interneurons that mediate PEI. These will be interesting questions for future research.

\section{APPENDIX}

\section{Relative reductions of $\boldsymbol{V}_{\text {contra }}$ and $\boldsymbol{V}_{\text {prox }}$}

The reduction of $V_{\text {contra }}$ is estimated here with reference to the diagrams of Figure 9, $A$ and $B$ (also see Vu et al., 1993). If the commissural resistance $R_{\text {com }}$ is substantially greater than the initial segment resistances $R_{\text {prox }}$ and $R_{\text {contra }}$, then the voltage $V_{\text {prox }}$ produced by a current $I$ injected ipsilateral to the recording electrode is approximately $I \cdot R_{\text {prox }}$, and the voltage $V_{\text {contra }}$ produced by a current injected contralaterally is $I \cdot\left(R_{\text {prox }} \cdot\right.$ $\left.R_{\text {contra }} / R_{\text {com }}\right)$. From this it follows that fractional drops, $\Delta V / V$, in voltages caused by recurrent or PEI can be expressed by:

$$
\frac{\Delta V_{\text {prox }}}{V_{\text {prox }}}=\frac{\Delta R_{\text {prox }}}{R_{\text {prox }}}
$$

and

$$
\frac{\Delta V_{\text {contra }}}{V_{\text {contra }}}=\frac{\Delta R_{\text {prox }}}{R_{\text {prox }}}+\frac{\Delta R_{\text {contra }}}{R_{\text {contra }}}-\frac{\Delta R_{\text {prox }}}{R_{\text {prox }}} \cdot \frac{\Delta R_{\text {contra }}}{R_{\text {contra }}}
$$

where $\Delta R_{\text {prox }}$ and $\Delta R_{\text {contra }}$ are the changes, caused by inhibition, in $R_{\text {prox }}$ and $R_{\text {contra }}$, respectively.

At the peak of PEI produced by stimulating a second root ipsilateral to the recording and current-passing electrodes, $\Delta V_{\text {prox }} / V_{\text {prox }}$ and hence $\Delta R_{\text {prox }} / R_{\text {prox }}$ (Eq. 1) were $0.11 \pm 0.01$ SEM (average of 13 preparations), and $\Delta R_{\text {contra }} / R_{\text {contra }}$ was $\sim 0.09 \pm 0.01$, as determined by second root stimulation contralateral to recording and current passing electrodes (two preparations). Given the small values of $\Delta R_{\text {prox }} / R_{\text {prox }}$ and $\Delta R_{\text {contra }} / R_{\text {con }}$ tra, the product on the right of Equation 2 is negligible compared with the sum on the left. Therefore, because $\Delta R_{\text {contra }} / R_{\text {contra }}$ is no greater than $\Delta R_{\text {prox }} / R_{\text {prox }}, \Delta V_{\text {contra }} / V_{\text {contra }}$ should not be more than twice $\Delta V_{\text {prox }} / V_{\text {prox }}$.

\section{REFERENCES}

Edwards DH (1990) Mechanisms of depolarizing inhibition at the crayfish giant motor synapse. I. Electrophysiology. J Neurophysiol 64:532-540.

Edwards DH, Heitler WJ, Leise EM, Fricke RA (1991) Postsynaptic modulation of rectifying electrical synaptic inputs to the LG escape command neuron in crayfish. J Neurosci 11:2117-2129.

Fischer TM, Carew TJ (1993) Activity-dependent potentiation of recurrent inhibition: a mechanism for dynamic gain control in the siphon withdrawal reflex of Aplysia. J Neurosci 13:1302-1314.

Fischer TM, Carew TJ (1995) Cutaneous activation of the inhibitory L30 interneurons provides a mechanism for regulating adaptive gain control in the siphon withdrawal reflex of Aplysia. J Neurosci 15:762-773.

Freund TF, Antal M (1988) GABA-containing neurons in the septum control inhibitory interneurons in the hippocampus. Nature 336:170-173.

Giaume C, Kado RT, Korn H (1987) Voltage-clamp analysis of a crayfish rectifying synapse. J Physiol (Lond) 386:91-112.

Jaslove SW, Brink PR (1986) The mechanism of rectification at the electrotonic motor giant synapse of the crayfish. Nature 323:63-65.

Johnson GE (1924) Giant nerve fibers in crustaceans with special reference to Cambarus and Palaemonetes. J Comp Neurol 36:19-33.

Kennedy D (1971) Crayfish interneurons. Physiologist 14:5-30.

Kennedy D, Calabrese R, Wine JJ (1974) Presynaptic inhibition: primary afferent depolarization in crayfish neurons. Science 186:451-454.

Kennedy D, McVittie J, Calabrese R, Fricke RA, Craselius W, Chiapella P (1980) Inhibition of mechanosensory interneurons in the crayfish. I. Presynaptic inhibition from giant fibers. J Neurophysiol 43:1495-1509.

Kirk MD (1985) Presynaptic inhibition in the crayfish CNS: pathways and synaptic mechanisms. J Neurophysiol 54:1305-1325.

Kirk, MD, Wine JJ (1984) Identified interneurons produce both primary afferent depolarization and presynaptic inhibition. Science 225:854-856.

Krasne FB (1969) Excitation and habituation of the crayfish escape reflex: the depolarizing response in lateral giant fibers of the isolated abdomen. J Exp Biol 50:29-46.

Krasne FB, Lee SC (1988) Response-dedicated trigger neurons as control points for behavioral actions: selective inhibition of lateral giant command neurons during feeding in crayfish. J Neurosci 8:3703-3712.

Krasne FB, Roberts A (1967) Habituation of the crayfish escape response during release from inhibition induced by picrotoxin. Nature 215:769-770.

Krasne FB, Teshiba TM (1995) Habituation of an invertebrate escape reflex due to modulation by higher centers rather than local events. Proc Natl Acad Sci USA 92:3362-3366.

Krasne FB, Wine JJ (1975) Extrinsic modulation of crayfish escape behavior. J Exp Biol 63:433-450.

Krasne FB, Wine JJ (1984) The production of crayfish tailflip escape responses. In: Neural mechanisms of startle behavior (Eaton R, ed), pp 179-211. New York: Plenum.

Krasne FB, Wine JJ (1987) Evasion responses of the crayfish. In: Aims and methods in neuroethology (Guthrie DM, ed), pp 10-45. Manchester, UK: Manchester UP.

Lee SC, Krasne FB (1993) Ultrastructure of the circuit providing input to the crayfish lateral giant neurons. J Comp Neurol 327:271-288.

Marder E, Paupardin-Tritsch D (1980) Picrotoxin block of a depolarizing Ach response. Brain Res 181:223-227.

Miller MW, Vu ET, Krasne FB (1992) Cholinergic transmission at the first synapse of the circuit mediating the crayfish lateral giant escape reaction. J Neurophysiol 68:2174-2184.

Mulloney B, Avecedo LD, Bradbury AG (1987) Modulation of the crayfish swimmeret rhythm by octopamine and the neuropeptide proctolin. J Neurophysiol 58:584-597.

Pennartz CM, Kitai ST (1991) Hippocampal inputs to identified neurons in an in vitro slice preparation of the rat nucleus accumbens: evidence for feed-forward inhibition. J Neurosci 11:2838-2847.

Roberts AM (1968) Recurrent inhibition in the giant-fibre system of the crayfish and its effect on the excitability of the escape response. J Exp Biol 48:545-567.

Shepherd GM (1990) The synaptic organization of the brain. New York: Oxford.

Sigvardt KA, Hagiwara G, Wine JJ (1982) Mechanosensory integration in the crayfish abdominal nervous system: structural and physiological 
differences between interneurons with single and multiple spike initiating sites. J Comp Physiol 148:143-157.

Tomasulo RA, Levy WB, Steward O (1991) LTP-associated EPSP/spike dissociation in the dentate gyrus: GABAergic and non-GABAergic components. Brain Res 561:27-34.

Trudeau LE, Castellucci VF (1993a) Sensitization of the gill and siphon withdrawal reflex of Aplysia: multiple sites of change in the neuronal network. J Neurophysiol 70:1210-1220.

Trudeau LE, Castellucci VF (1993b) Functional uncoupling of inhibitory interneurons plays an important role in short-term sensitization of Aplysia gill and siphon withdrawal reflex. J Neurosci 13:2126-2135.

Turner DA (1990) Feed-forward inhibitory potentials and excitatory interactions in guinea-pig hippocampal pyramidal cells. J Physiol (London) 422:333-350.

Van Harreveld A (1936) A physiological solution for freshwater crustacea. Proc Soc Exp Biol Med 34:428-432.

Vu ET, Krasne FB (1992) Evidence for a computational distinction between proximal and distal neuronal inhibition. Science 255:1710-1712.

Vu ET, Krasne FB (1993) Crayfish tonic inhibition: prolonged modulation of behavioral excitability by classical GABAergic inhibition. J Neurosci 13:4394-4402.

Vu ET, Lee SC, Krasne FB (1993) The mechanism of tonic inhibition of crayfish escape behavior: distal inhibition and its functional significance. J Neurosci 13:4379-4393.

Watanabe A, Grundfest H (1961) Impulse propagation at the septal and commissural junctions of crayfish lateral giant axons. J Gen Physiol 45:267-308.

Wine JJ (1984) The structural basis of an innate behaviour pattern. J Exp Biol 112:283-319.

Wine JJ, Krasne FB (1972) The organization of escape behavior in the crayfish. J Exp Biol 56:1-18.

Zucker RS (1972) Crayfish escape behavior and central synapses. II. Physiological mechanisms underlying behavioral habituation. J Neurophysiol 35:599-651. 